University of Nebraska - Lincoln

DigitalCommons@University of Nebraska - Lincoln

USGS Staff -- Published Research

US Geological Survey

5-28-2018

\title{
Macrobenthic infaunal communities associated with deep-sea hydrocarbon seeps in the northern Gulf of Mexico
}

Travis W. Washburn

Harte Research Institute, Texas A\&M University-Corpus Christi,, twashburn@tamucc.edu

Amanda W. J. Demopoulos

Southeast Ecological Science Center, United States Geological Survey

Paul A. Montagna

Harte Research Institute, Texas A ${ }^{\prime} M$ University-Corpus Christi,

Follow this and additional works at: http://digitalcommons.unl.edu/usgsstaffpub

Part of the Geology Commons, Oceanography and Atmospheric Sciences and Meteorology Commons, Other Earth Sciences Commons, and the Other Environmental Sciences Commons

Washburn, Travis W.; Demopoulos, Amanda W. J.; and Montagna, Paul A., "Macrobenthic infaunal communities associated with deep-sea hydrocarbon seeps in the northern Gulf of Mexico" (2018). USGS Staff -- Published Research. 1072.

http://digitalcommons.unl.edu/usgsstaffpub/1072

This Article is brought to you for free and open access by the US Geological Survey at DigitalCommons@University of Nebraska - Lincoln. It has been accepted for inclusion in USGS Staff -- Published Research by an authorized administrator of DigitalCommons@University of Nebraska - Lincoln. 
Supplementary Table S1. Data for stations used in this study. A) All stations sampled from 2009 - 2013 used to explore macrobenthic communities at natural hydrocarbon seeps in this study. B) All background stations sampled during DGoMB cruises from $2000-$ 2002 and DWH response cruises in 2010. Rep $=$ replicate, $\mathrm{PC}=$ pushcore, $\mathrm{MC}=$ multicore, $\mathrm{BC}=$ boxcore, and $\#$ of Reps $=$ the number of cores collected for macrofaunal analysis at each DGoMB and DWH station.

A)

\begin{tabular}{lllllllll}
\hline Station & Year & Rep & Latitude & Longitude & Depth & Seep & Habitat & Method \\
\hline DC583 & 2009 & 1 & 28.3856 & 87.3886 & 2461 & Background & Background & PC \\
DC583 & 2009 & 2 & 28.3855 & 87.3885 & 2449 & Near & Tubeworms & PC \\
DC583 & 2009 & 3 & 28.3849 & 87.3883 & 2447 & Near & Tubeworms & PC \\
DC583 & 2009 & 4 & 28.3855 & 87.3884 & 2452 & Background & Background & PC \\
DC583 & 2009 & 5 & 28.3855 & 87.3885 & 2449 & Near & Tubeworms & PC \\
\hline DC673 & 2010 & 1 & 28.31 & 87.311 & 2601 & Near & Tubeworms & PC \\
DC673 & 2010 & 2 & 28.31 & 87.311 & 2601 & Near & Tubeworms & PC \\
DC673 & 2010 & 3 & 28.31 & 87.311 & 2601 & Near & Tubeworms & PC \\
GC246 & 2010 & 1 & 27.7016 & 90.6486 & 834 & Near & Microbial mat & PC \\
GC246 & 2010 & 2 & 27.702 & 90.649 & 834 & Near & Microbial mat & PC \\
GC354 & 2010 & 1 & 27.598 & 91.823 & 567 & Near & Tubeworms & PC \\
GC354 & 2010 & 2 & 27.5977 & 91.827 & 527 & Background & Background & PC \\
GC354 & 2010 & 3 & 27.598 & 91.823 & 567 & Near & Tubeworms & PC \\
GC354 & 2010 & 4 & 27.5981 & 91.8231 & 567 & Near & Tubeworms & PC \\
GC354 & 2010 & 5 & 27.5977 & 91.827 & 527 & Background & Background & PC \\
GC354 & 2010 & 6 & 27.598 & 91.827 & 527 & Background & Background & PC \\
\hline GC600-1 & 2012 & 1 & 27.3644 & 90.5643 & 1263 & Near & Seep & PC \\
GC600-1 & 2012 & 2 & 27.3644 & 90.5643 & 1263 & Near & Seep & PC \\
GC600-2 & 2012 & 1 & 27.3698 & 90.5712 & 1221 & Near & Microbial mat & PC \\
GC600-2 & 2012 & 2 & 27.3698 & 90.5712 & 1221 & Near & Microbial mat & PC \\
GC600-2 & 2012 & 3 & 27.37 & 90.571 & 1220 & Near & Microbial mat & PC \\
GC600-3 & 2012 & 1 & 27.3728 & 90.5749 & 1181 & Near & Seep & MC
\end{tabular}




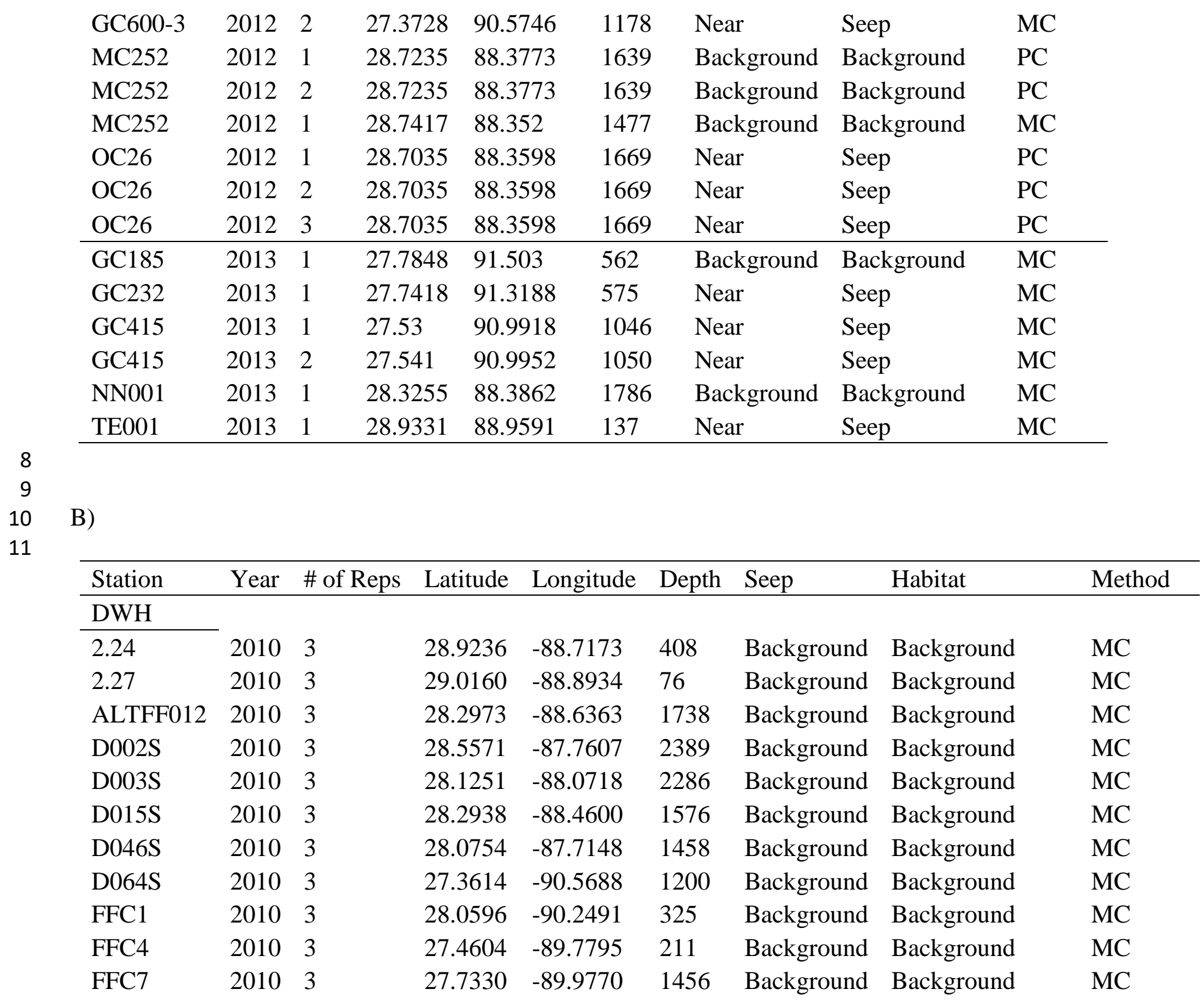




\begin{tabular}{lllllllll} 
FFMT1 & 2010 & 1 & 28.5396 & -89.8288 & 1015 & Background & Background & MC \\
M011S & 2010 & 3 & 28.0004 & -88.8800 & 1700 & Background & Background & BC \\
S01S & 2010 & 2 & 29.2533 & -88.6907 & 65 & Background & Background & BC \\
\cline { 1 - 3 } DGoMB & & & & & & & & \\
C4 & 2000 & 2 & 27.4563 & -89.7744 & 1455 & Background & Background & BC \\
C7 & 2000 & 2 & 27.7306 & -89.9784 & 1080 & Background & Background & BC \\
MT1 & 2000 & 3 & 28.5408 & -89.8269 & 487 & Background & Background & BC \\
NB2 & 2000 & 1 & 27.1337 & -91.9993 & 1530 & Background & Background & BC \\
S37 & 2000 & 1 & 28.5567 & -87.7622 & 2388 & Background & Background & BC \\
S38 & 2000 & 1 & 28.2719 & -87.3252 & 2635 & Background & Background & BC \\
WC12 & 2000 & 1 & 27.3232 & -91.5558 & 1168 & Background & Background & BC \\
WC5 & 2000 & 1 & 27.7713 & -91.7621 & 400 & Background & Background & BC \\
BH & 2001 & 1 & 27.798 & -91.4703 & 546 & Background & Background & BC \\
C7 & 2001 & 1 & 27.7299 & -89.9817 & 1072 & Background & Background & BC \\
MT1 & 2001 & 1 & 28.5381 & -89.8277 & 490 & Background & Background & BC \\
MT1 & 2002 & 2 & 28.558 & -89.822 & 465 & Background & Background & BC \\
\hline
\end{tabular}


Supplementary Table S1. Benthic community characteristics. The average abundance ( $\mathrm{n} \mathrm{m}^{-2}$ ), richness (R), diversity (Hill's N1), and evenness $\left(\mathrm{J}^{\prime}\right)$ of macrobenthos at individual seep sites. Standard errors are given in parentheses. The number of cores for each calculation is given. These parameters were also averaged for all seep samples across depths $(<1000,1000-2000$, and $>2000 \mathrm{~m})$, seep habitats (Microbial Mat, Soft-bottom Seep, and Tubeworm), and collection method (Multicore and pushcore). Similarity and multivariate dispersion (MVDISP) were also included.

\begin{tabular}{|c|c|c|c|c|c|c|c|}
\hline Seep Station & $\mathrm{N}$ & Abundance & $\mathrm{R}$ & N1 & $\mathrm{J}^{\prime}$ & $\%$ similarity & MVDISP \\
\hline DC583-09 & 3 & $2421(278)$ & $4.7(0.33)$ & $4.18(0.38)$ & $0.93(0.02)$ & 31.7 & 1.667 \\
\hline GC246-10 & 2 & $40892(16577)$ & $7.0(4.00)$ & $3.41(1.00)$ & $0.71(0.09)$ & 26.0 & 1.8 \\
\hline GC354-10 & 3 & $25156(3738)$ & $13.0(1.73)$ & $9.29(1.14)$ & $0.87(0.01)$ & 48.4 & 1 \\
\hline DC673-10 & 3 & $4526(862)$ & $8.7(0.67)$ & $7.65(0.64)$ & $0.94(0.02)$ & 55.1 & 0.833 \\
\hline GC600-12-1 & 2 & $17525(1105)$ & $9.0(0)$ & $4.03(0.53)$ & $0.63(0.06)$ & 64.3 & 0.3 \\
\hline GC600-12-2 & 3 & $17262(12055)$ & $7.3(1.76)$ & $4.51(1.21)$ & $0.77(0.12)$ & 60.4 & 0.567 \\
\hline GC600-12-3 & 2 & $9475(63)$ & $16.5(0.5)$ & $9.93(1.12)$ & $0.82(0.05)$ & 50.0 & 1.4 \\
\hline OC26-12 & 3 & $5052(365)$ & $9.7(0.67)$ & $8.33(0.95)$ & $0.93(0.03)$ & 54.3 & 0.8 \\
\hline GC232-13 & 1 & 5146 & 15.0 & 10.46 & 0.87 & NA & NA \\
\hline GC415-13 & 2 & $4142(126)$ & $10.5(1.5)$ & $7.27(0.63))$ & $0.85(0.01)$ & 52.2 & 0.9 \\
\hline TE001-13 & 1 & 3640 & 10.0 & 6.37 & 0.80 & NA & NA \\
\hline \multicolumn{8}{|l|}{ Depth } \\
\hline$>2000 \mathrm{~m}$ & 6 & $3473(623)$ & $6.7(0.95)$ & $5.92(0.84)$ & $0.93(0.01)$ & 43.4 & 0.733 \\
\hline $1000-2000 \mathrm{~m}$ & 12 & $10769(3117)$ & $10.3(1.00)$ & $6.75(0.76)$ & $0.81(0.04)$ & 56.7 & 0.956 \\
\hline$<1000 \mathrm{~m}$ & 7 & $23720(6862)$ & $11.1(1.62)$ & $7.36(1.22)$ & $0.81(0.03)$ & 42.8 & 1.075 \\
\hline \multicolumn{8}{|l|}{ Habitat } \\
\hline Tubeworm & 9 & $10701(3793)$ & $8.8(1.32)$ & $7.04(0.85)$ & $0.91(0.02)$ & 45.0 & 1.145 \\
\hline Soft-bottom & 11 & 7839 (1572) & $11.5(0.94)$ & $7.66(0.72)$ & $0.82(0.03)$ & 54.9 & 0.937 \\
\hline Microbial Mat & 5 & $26714(10227)$ & $7.2(1.59)$ & $4.07(0.78)$ & $0.74(0.7)$ & 51.8 & 0.824 \\
\hline \multicolumn{8}{|l|}{ Method } \\
\hline Multicorer & 6 & 6003 (1117) & $13.2(1.35)$ & $8.54(0.79)$ & $0.83(0.02)$ & 51.1 & 0.525 \\
\hline Pushcore & 19 & $14741(3542)$ & $8.5(0.75)$ & $6.15(0.60)$ & $0.84(0.03)$ & 49.4 & 1.042 \\
\hline All Stations & 25 & $12644(2792)$ & $9.6(.076)$ & $6.72(0.53)$ & $0.84(0.02)$ & 25.0 & \\
\hline
\end{tabular}


